\title{
Innovación y enseñanza. De la "moda" de innovar a la transformación de la práctica docente
}

\author{
Inovação e ensino. Da "moda" de inovar à transformação da prática de ensino
Innovation and teaching. From the "fashion" of innovation to the transformation of teaching practice

\begin{abstract}
JUANA M. SANCHO-GIL*
$\diamond$

RESUMEN

El mundo está constituido por un conjunto de cambios permanentes y el ámbito de la educación no es una excepción. Este artículo comienza situando las (in)constancias del cambio, sus derivas y paradojas y sigue con un breve apunte sobre la evolución de las ideas educativas, para plantear los desafíos que supone el imperativo de la innovación en el momento actual. A partir de ahí, se argumenta que ir más allá de la innovación como "moda", requiere una profunda transformación de la práctica, para lo que se precisa tener en cuenta cinco elementos clave: quiénes son los docentes y estudiantes, cómo aprenden y cuáles son sus necesidades; qué tipo de conocimiento se considera importante enseñar para que al alumnado le resulte personal y socialmente valioso; qué tecnologías educativas se tendrían que utilizar o desarrollar para contribuir a alcanzar las finalidades planteadas; qué experiencias de aprendizaje habría que implementar para promover la implicación de los estudiantes y el sentido de aprender; qué sistemas evaluación se necesitan para dar cuenta del aprendizaje realizado.
\end{abstract}

Palabras clave: Cambio educativo. Currículo. Desarrollo educativo. Tecnología educativa. Evaluación.

\section{RESUMO}

O mundo é constituído por um conjunto de mudanças permanentes, e o campo da educação não é uma exceção. Este artigo começa por situar as (in)constantes mudanças, suas derivações e paradoxos, e continua com uma breve nota sobre a evolução das ideias educacionais, para elevar os desafios que o imperativo da inovação no momento presente supõe. A partir daí, argumenta-se que ir além da inovação como "moda" exige uma transformação profunda da prática, para a qual é necessário levar em consideração cinco elementos-chave: quem são professores e alunos, como aprendem e quais são suas necessidades; que tipo de conhecimento é considerado importante ensinar para que os alunos o percebam como pessoalmente e socialmente valioso; quais tecnologias educacionais devem ser usadas ou desenvolvidas no sentido de contribuir para alcançar os objetivos declarados; quais experiências de aprendizagem são convenientes implementar para promover o envolvimento dos alunos e seu senso de aprendizagem; quais sistemas de avaliação são necessários para explicar o aprendizado alcançado.

Palavras-chave: Mudança educacional. Currículo. Desenvolvimento educacional. Tecnologia educacional. Avaliação.

\begin{abstract}
The world is made up of a set of permanent changes and the field of education is no exception. This article begins by situating the (in)constancy of change, its drifts and paradoxes and continues with a brief note on the evolution of educational ideas, to raise the challenges posed by the imperative of innovation at the present time. From this point on, it is argued that going beyond innovation as "fashion" requires a profound transformation of educational practice, for which it is necessary to take into account five key elements: who teachers and students are, how they learn and what their needs are; what kind of knowledge is considered important to teach in order to make it personal and socially valuable for the students; what educational technologies should be used or developed to contribute to achieving the goals set; what learning experiences should be implemented to promote students' engagement and the meaning of learning; what assessment systems are needed to account for the learning undertaken.
\end{abstract}

Keywords: Educational change. Curriculum. Educational development. Educational technology. Evaluation.

\footnotetext{
* Coordinadora del grupo de investigación consolidado ESBRINA - Subjetividades, visualidades y entornos educativos contemporáneos $<$ http://esbrina.eu $>$ y de REUNI+D - Red de investigación e innovación educativa. Cambios sociales y retos para la educación en la era digital. MINECO $<$ http://reunid.eu $>$. Miembro de INDAGA-T - Grupo de innovación docente para favoreces la indagación. Universidad de Barcelona. <http://www.ub.edu/indagat/>. E-mail:jmsancho@ub.edu
} 


\section{LA (IN)CONSTANCIA DEL CAMBIO}

Una mirada atenta a nuestro alrededor nos permite verificar los constantes procesos de cambio en la naturaleza, la sociedad, la cultura, la tecnología, las personas y nosotros mismos. No perece haber nada más constante que el cambio. Lo que en un momento nos parece que algo "es" de una manera, al poco tiempo puede haberse convertido en algo diferente. Esta constatación llevó a místicos como Siddhārtha Gautama (Buda) a afirmar que todo cambia y que nada permanece sin cambio. Y a filósofos como Georg W. F. Hegel a plantear, para fundamentar su noción de dialéctica, que lo que nos permite desarrollar el conocimiento es lo que nos contradice. Lo que nos lleva a cuestionar algo que consideramos "verdadero" o "evidente" y nos posibilita hacer nuevas preguntas. Para ello, el filósofo alemán, en su obra Ciencia de la lógica (1812-1816) proponía plantearse una tarea tan sencilla como escribir durante la mañana algo tan evidente como "ahora es de día". Después, dejar pasar varias horas y esa "verdad" irrefutable se ha convertido en una falsedad. Lo que no significa que lo fuera cuando se escribió, sino que la realidad es cambiante y en está hecha de oposiciones.

En nuestro entorno cotidiano, personal y profesional, podemos encontrar numerosos ejemplos de todo lo que ayer era "cierto" y lo que hoy ya no se sustenta. De que lo que ayer "funcionaba" ahora ya no sirve. En el campo específico de la educación, lo que en un momento dado se consideraba "una buena Escuela", "un buen docente", "una buena educación", en otro puede ser visto como algo inadecuado por no tener en cuenta las características y necesidades cambiantes de la sociedad.

En los últimos cuarenta años, la aceleración de los cambios políticos, sociales y económicos de la mano del desarrollo de las Tecnologías de la Información y Comunicación (TIC), han llevado a autores como Peter Senge, a argumentar que, en este contexto, las instituciones "capaces de aprender" serán las únicas que sobrevivirán. Desde su perspectiva, "las organizaciones que realmente sobresaldrán en el futuro serán las que descubran cómo conseguir el compromiso y la capacidad de aprender de todos $^{1}$ los niveles de una organización" (SENGE, 1990, p.3-4). Sin embargo, cuanto más grandes, asentadas y burocratizadas están las instituciones, más difícil resulta transformarlas (BELASCO, 1992). De entre ellas, las que parece particularmente arduo cambiar son las del ámbito de la educación (SARASON, 1990; BRENNAN; SHAH, 2011; SWANGER, 2016).

Puede sorprender que las instituciones responsables de la educación y, por tanto, vinculadas al pensamiento y

\footnotetext{
1 Énfasis de la autora.
}

el conocimiento, sean las que acumulen una mayor fuerza inercial. La explicación la encontramos en distintos ángulos. En uno de ellos se sitúa la idea de FOUCAULT (1994) de que, con el tiempo, los sistemas - en este caso el educativo, se convierten en poderosos dispositivos organizativos entendidos como

un conjunto decididamente heterogéneo que componen los discursos, las instituciones, las habilitaciones arquitectónicas, las decisiones reglamentarias, las leyes, las medidas administrativas, los enunciados científicos, las proposiciones filosóficas, morales, filantrópicas. En fin, entre lo dicho y lo no dicho, he aquí los elementos del dispositivo. El dispositivo mismo es la red que tejemos entre estos elementos. [...] Por dispositivo entiendo un tipo, diríamos, de formación que, en un momento dado, ha tenido por función mayoritaria responder a una urgencia. De este modo, el dispositivo tiene una función estratégica dominante [...]. He dicho que el dispositivo tendría una naturaleza esencialmente estratégica; esto supone que allí tiene lugar una cierta manipulación de relaciones de fuerza, ya sea para desarrollarlas en tal o cual dirección, ya sea para bloquearlas, o para estabilizarlas, utilizarlas. Así, el dispositivo siempre está inscrito en un juego de poder, pero también ligado a un límite o a los límites del saber, que le dan nacimiento, pero, ante todo, lo condicionan. Esto es el dispositivo: estrategias de relaciones de fuerza sosteniendo tipos de saber, y [son] sostenidas por ellos (p. 229 y ss.).

En este ángulo es fundamental considerar que uno de los elementos más "duros" de este dispositivo son los edificios, construidos en un momento y con un presupuesto dados $\mathrm{y}$, sobre todo, con una noción determinada de la educación (FOUCAULT, 1976; SANCHO, 2000; RIGOLON, 20132). La disposición de los espacios construidos influye en las prácticas pedagógicas a lo largo de los años, en general, de manera inercial.

En otro ángulo diferentes, pero totalmente interconectado, y no menos importante por su incidencia fundamental en la constitución a este fenómeno, se encuentra lo que podríamos llamar "la herida narcisista", "el miedo a la obsolescencia" o "el temor a perder el control". ¿A qué me refiero? Las personas con responsabilidad en los distintos niveles los sistemas escolares: macro (responsables de las políticas educativas, grupos con poder de influencia e intereses...), meso (gestores, inspectores...) y micro (directores de centro, profesores) pertenecen al grupo de los que han tenido éxito en sus estudios. De los que han sido capaces de saltar todas las vallas del sistema escolar. De ahí que, en

\footnotetext{
2 Ver el número monográfico de la monográfico de Bordón. Revista de Pedagogía, sobre arquitectura y educación. Disponible en: <https:// recyt.fecyt.es/index.php/BORDON/issue/view/2449>. Acceso en: 11 oct. 2016.
} 
general, tiendan a pensar que "lo que les fue bien ellos, les ha de ir bien al alumnado actual". Por otra parte, como argumentaba Lawrence Stenhouse, las personas son malas implementadoras de las ideas de otros, por lo que si, sin haber contado con ellas, ni tenido en cuenta sus condiciones laborales, se sienten "obligadas" a introducir cambios, pueden sentirse desautorizadas, menospreciadas y fuera de contexto. La enseñanza es una profesión que implica una alta carga emocional (SANCHO; HERNÁNDEZ, 2016). Un docente tiende a pensar que siempre hace "lo mejor". De ahí que, si no se cuenta con la actitud dialéctica de la hablaba Hegel, la introducción impuesta de cualquier cambio se tienda a interpretar como una evaluación negativa de la práctica. Esto no sucede solamente en el nivel micro, sino que gestores y responsables de políticas educativas pueden sufrir el mismo tipo estrés. Lo ilustraré con un ejemplo.

Hace unos años, con el profesor Fernando Hernández, participamos en los estudios previos y la primera fase de planificación de una reforma del sistema escolar de un país la antigua URSS. Desde el principio, en nuestra área de intervención: la formación permanente del profesorado, implicamos al máximo de personas posibles de los distintos niveles de sistema. Tanto valoraciones como propuestas se hacían de forma contextual y con el máximo de consenso posible. El proyecto marchaba más que razonablemente bien, la mayoría de las personas sentían que se necesitaba un cambio profundo en todo el sistema $\mathrm{y}$, en particular, en la formación permanente del profesorado. Pero sentíamos algunos focos de resistencia. Sobre todo, en el nivel meso, en el organismo que tradicionalmente se venía ocupando de esa formación. Después de múltiples conversaciones con los responsables, logramos que verbalizasen el problema. Para ellos, si se introducía un modelo de formación permanente más distribuido y basado en las necesidades reales del profesorado y las esuelas, significaba que lo que ellos habían estado haciendo "estaba mal". En este punto, Hegel nos iluminó. Así que les propusimos lo siguiente: "Cuando ustedes tenían 18 años, les compraron un traje precioso, del que disfrutaron durante un tiempo, pero hoy, 'por todos los cambios experimentados en su cuerpo, les resulta totalmente inadecuado. Significa que su traje no era precioso o ya no lo es, o que el sastre que lo elaboró no fuese bueno? Por supuesto que no, lo único que significa es que sus necesidades actuales son otras". Esta conversación significó un punto de inflexión.

Desde las tensiones que genera la inevitabilidad de los cambios y las dificultades de las instituciones para cambiar, en este artículo, en primer lugar, se abordan las corrientes constantes de pensamiento innovador en el campo de la educación. Y, en segundo lugar, el conjunto de dimensiones a tener en cuenta para que la necesidad de innovar no se convierta en una 'moda', sino en una transformación paulatina, sostenible y con sentido de la práctica educativa y las finalidades de la educación.

\section{LA EVOLUCUIÓN DE LAS IDEAS EDUCATIVAS}

Los libros de historia de la educación recogen el conjunto de ideas y propuestas, en su momento innovadoras, desarrolladas por distintos autores y más o menos implementadas en los distintos niveles de los sistemas de escolares. Proposiciones y proyectos que suelen elaborarse para responder a los cambiantes contextos políticos, sociales, culturales y económicos. En este sentido, Robert MCCLINTOCK (1993), para argumentar la necesidad de transformar las ideas base de la educación en la era de las TIC, establecía una estrecha conexión entre la aparición de la imprenta y la reforma educativa a comienzos de la Edad Moderna, centrándose en: Erasmo de Rotterdam (1466-1536) - Enseñanza basada en libro de texto; Lutero (14831546) - Participación cultural a través de las letras; Elyot (1490-1546) - Textos diseñados para popularizar los conocimientos más útiles; Melanchton (1497-1560) - Avance por grados basado en la demostración de los logros obtenidos; Loyola (1491-1566) - Motivación competitiva; Sturn (1507-1589) - Agrupación por edades según una secuencia curricular; Ramus (1515-1572) División de los temas en secuencias; Ascham (1515-1568) - Libros de texto y manuales de autodidacta; Mulcaster (1530-1611) - Los profesores tienen que adaptarse a las diferencias individuales; Ratke (1571-1636) - Uso de la lengua vernácula y de la experiencia controlada en la enseñanza; Comenio (1592-1640): Diseño didáctico de asociación de ideas con imágenes.

Del mismo modo que la trasformación de las sociedades agrícolas en industriales, el reconocimiento del derecho a la educación de personas tradicionalmente excluidas de este privilegio/derecho, los nuevos lenguajes (sobre todo el visual), las nuevas miradas sobre el aprendizaje y las maneras de representar el conocimiento sustentaron el pensamiento y la práctica educativa de autores como Dewey (1859-1953) - la educación como experiencia vital; Steiner (1861-1925) - Antroposofía: el legado intelectual, el legado artístico y el legado disciplinar; Montessori (1870-1952) - actividad dirigida por el aprendiz y observación clínica por parte del docente; Kilpatrick (1871-1965) - método de proyectos; Decroly (1871-1932) - método sustentado en las necesidades e intereses de la infancia; Freinet (1896-1966) - el profesorado ha de poner a disposición del alumnado las técnicas más apropiadas; Freire (1921-1997) - quien enseña aprende y quien aprende enseña al aprender; 
Stenhouse (1926-1982) - el currículo como hipótesis de acción. Durante el siglo XX, además los diferentes movimientos de renovación pedagógica propulsados por el propio profesorado (HERNÁNDEZ, J. M, 2011), con el asentamiento de los sistemas escolares en la mayoría de los países y el acceso a la educación, no solo fundamental sino también superior, de capas cada vez más amplias de la educación, también han conllevado un conjunto de reformas sucesivas, que no siempre han conseguido los objetivos propuestos (SANCHO, 1998a; SARASON, 1990). En este contexto, los distintos estudios llevados a cabo desde la perspectiva del cambio educativo, han puesto de manifiesto la complejidad de los elementos implicados en la mejora de la educación (FULLAN, 2002; SANCHO, 2000; STOLL; FINK, 1999; STOLL; FINK; EARL, 2004).

En estos momentos, en plena sociedad postindustrial y digital y con el aumento de los movimientos neoconservadores, se acrecientan los discursos relativos a la necesidad de transformar profundamente los sistemas escolares, desde la educación infantil a la postuniversitaria, desde perspectivas muy diferentes (ADAMS BECKER et al., 2017; BIESTA, 2015; BUXARRAIS; MARTÍNEZ, 2015; EDU-FACTORY Y UNIVERSIDAD NÓMADA, 2010; MONTGOMERY; KEHOE, 2016; SANCHO; HERNÁNDEZ, F., 2006). De ahí la necesidad de abordar la innovación desde una perspectiva compleja y transformadora.

\section{EL IMPERATIVO DE LA INNOVACIÓN}

En los últimos tres años, en Cataluña se está produciendo una eclosión de iniciativas innovadoras, sobre todo en los ámbitos de la educación infantil, primaria y secundaria. Junto a propuestas con más trayectoria como las vinculadas al aprender mediante proyectos (ANGUITA et al., 2010; AUTORÍA COMPARTIDA, 2016; HERNÁNDEZ, F.; VENTURA, 2008) o los distintos movimientos de renovación pedagógica, han comenzado a emerger un conjunto de acciones sin precedentes. En 2014, algunas escuelas de los Jesuitas comenzaron a implementar el proyecto Horizonte $2020^{3}$, basado en una perspectiva centrada en el alumnado, el trabajo por proyectos, la flexibilidad y el aprendizaje autónomo. Como en el caso de la reforma puesta en marcha en Finlandia en 2016, previamente experimentada en distintos centros (JUUSO et al., 2014), la remodelación de los espacios de aprendizaje representa un papel fundamental.

En 2016, aparece la denominada Escola Nova 21, mediante un convenio del centro UNESCO de Cataluña,

\footnotetext{
${ }_{3}$ Disponible en: $<$ http://h2020.fje.edu/es $>$. Acceso en: 2 dic. 2017
}

con una fundación privada, la obra social de un banco y una universidad privada, que se propone una transformación del sistema educativo. En lo que han dado en llamar un "marco de escuela avanzada", pretenden posibilitar muchos modelos y proyectos diferentes pero que tengan en común: un propósito educativo que dé respuesta a los retos actuales, unas prácticas basadas en el conocimiento existente, y una evaluación y una organización al servicio del aprendizaje $\mathrm{e}^{4}$. Es este momento, más de 450 escuelas de enseñanza infantil, primaria y secundaria están vinculadas a esta iniciativa.

Durante el curso 2016-2017, dos colaboradoras de mi grupo de investigación ${ }^{5}$, identificaron, en Cataluña, unas 50 redes que, de manera más o menos activa, se planteaban el tema de la innovación educativa. Solo 10 de ellas parecían relativamente vinculadas o apoyadas por el Departamento de Enseñanza de la Gobierno de Cataluña.

En este contexto, han proliferado los actos y las publicaciones sobre el tema de la innovación ${ }^{6}$. De ahí la importancia de la noción de innovación a la que nos referimos. Se trata de un concepto industrial, relacionado con el capitalismo de consumo intenso que necesita producir cada temporada un nuevo producto para que la gente consuma, lo necesite o no? En este caso, lo que preocupa es "estar a la moda", lo que conlleva estar inmediatamente demodée, anticuado y constantemente pendiente de los "vendedores" de novedades.

O más bien nos referimos un posicionamiento que está pendiente de los cambios, cada vez más acelerados, que tienen lugar en nuestra sociedad y que implican nuevas demandas, necesidades y retos para la educación? Lo que esto implica es que hay que estar alerta, hay que entender los fenómenos que nos rodean, hay que analizar lo que tenemos que preservar, lo que tenemos que erradicar, lo que tenemos que transformar, lo que tenemos que mejorar, lo que tenemos que soñar y proyectar, y todo lo que ello conlleva. Hay que estar un proceso de mejora continua que no pasa de moda y nos permite seguir aprendiendo.

Si nos situamos en esta segunda noción, estamos hablando más de transformación educativa y en los siguientes apartados me referiré a las cinco dimensiones implicadas en el difícil y apasionante proceso de transformación de las instituciones de enseñanza y la práctica docente. Teniendo en cuanta que ésta no se da en el vacío, sino en un sistema complejo en el que los niveles micro, meso y macro necesitan ponerse a dialogar.

\footnotetext{
${ }^{4}$ Disponible en: <https://www.escolanova21.cat/castellano>. Acceso en: 3 abr. 2017.

5 Judit Vilarnau y Mònica Caro.

6 Ver el monográfico sobre Práctica, teoría y avaluación de la innovación de la Revista Catana de Pedagogia. Disponible en: <http://revistes. iec.cat/index.php/RCP/issue/view/9639/showToc $>$. Acceso en: 7 dic. 2017 y PALOU, Juli; FONS, Montserrat (Coord.). Escenaris nous, aprenentatges nous. Dossier Graó. Barcelona: Graó, 2016.
} 


\section{LOS CINCO ELEMENTOS IMPRESCINDIBLES}

Los sistemas escolares los componen personas - las más importantes de ellas docentes y estudiantes -, que han de enseñar y aprender conocimientos personal y socialmente valiosos, con las tecnologías más adecuadas, mediante experiencias de aprendizaje que tengan sentido y que han de poder calibrar la calidad de los procesos y los resultados. De ahí que un sistema escolar transformador tendría que deliberar en torno los siguientes puntos.

\section{EL PROFESORADO Y EL ALUMANO}

La primera y más pertinente cuestión consiste en preguntarse sobre quiénes son los docentes y los estudiantes. Tanto un colectivo como otro va cambiando a lo largo del tiempo y, en un proceso continuado de transformación, resulta fundamental preguntarse por sus saberes, expectativas, predisposiciones, formas de aprender, necesidades formativas, etc. Existen estudios sobre la infancia y la juventud actual y sus formas de aprender (HERNÁNDEZ, F., 2017; ITO et al., 2011; TWENGE, 2017, entre muchos otros). Además de informes periódicos como TALIS, estudio internacional de la enseñanza y el aprendizaje, realizado por la OCED, contamos también con investigación sobre el profesorado (SANCHO; HERNÁNDEZ, F., 2016, entre otros).

Por otra parte, aunque a menudo parece olvidarse, como argumenta PHILLIPS (2014, p. 10),

\begin{abstract}
El aprendizaje es un fenómeno que involucra a personas reales que viven en contextos sociales reales y complejos de los que no se pueden abstraer de manera significativa. [...] los aprendices están contextualizados. Pertenecen a un género, tienen una orientación sexual, un nivel socioeconómico, son parte de una etnia, de una cultura de origen; tienen intereses -y cosas que les aburren; pueden o no haber desayunado; y viven en barrios con o sin frecuente violencia armada o terremotos, les atrae (o se enfrentan con) la personalidad de sus docentes.
\end{abstract}

Y que el aprender con sentido implica un desafío para las estructuras del conocimiento y las creencias actuales, al permitirnos descubrir nuevos horizontes y hacernos nuevas preguntas. Tiene manifestaciones psicológicas físicas y requiere la estimulación del cerebro. Pero, además precisa de tiempo para la reflexión, la consolidación y la internalización y no está ligado a un tiempo y a un espacio (ERSTAD et al. 2016; HERNÁNDEZ, F., 2017, MIÑO, 2017; LIVINGSTONE; SEFTON-GREEN, 2016), algo que tiende a desconsiderar la presión espacial y temporal de las instituciones educativas. De ahí la importancia de centrar también la investigación en las formas de aprender de los docentes.
La constatación de esta necesidad, nos llevó a plantearnos el proyecto de investigación "APREN-DO - Cómo aprenden los docentes: implicaciones educativas y retos para afrontar el cambio social", que estamos desarrollando en estos momentos ${ }^{7}$. El foco del estudio es explorar con los docentes, qué, cómo, donde, con qué y con quien aprenden dentro y fuera de la escuela, así como las posibles consecuencias de su aprendizaje para la mejora de la educación y el cambio educativo. Mediante la utilización de métodos visuales, hemos desarrollado con 29 docentes de secundaria ${ }^{8}$ cartografías de su aprendizaje. Esto nos está permitiendo explorar "las sensaciones, intensidades y texturas a través de las cuales se experimenta el aprendizaje" (COLEMAN; RINGROSE, 2014, p. 4) y la multiplicidad de los mundos de aprendizaje de los docentes (MIÑO; ALONSO; BOSCO, et al., 2017).

En este punto, una cuestión fundamental estriba en plantearse hasta qué punto, los responsables de las políticas educativas, los distintos gestores y administradores, los responsables de la formación inicial y permanente del profesorado, las propios escuelas y los docentes analizan periódicamente y de forma contextual las características y necesidades de ambos colectivos.

\section{QUÉ SE ENTIENDE POR CONOCIMIENTO}

SAWYER (2008, p. 2) ofrece una clara síntesis de la noción de conocimiento que subyace en la mayoría de los sistemas escolares.

- El conocimiento es una colección de hechos sobre el mundo y de procedimientos para resolver problemas.

- La finalidad de la escuela es meter estos hechos y procedimientos en la cabeza del alumnado. Se considera educado a quien posee una buena colección de estos datos y procedimientos.

- El profesorado debe conoce estos hechos y procedimientos y su labor es trasmitírselos al alumnado.

- Los hechos y los procedimientos más simples han de aprenderse antes, para seguir con hechos y procedimientos más complejos. Esta definición de simplicidad y complejidad y la propia secuenciación de los materiales de enseñanza están determinados por el profesorado, los autores de los libros de texto o por expertos adultos (matemáticos,

\footnotetext{
7 Ministerio de Economía, Industria y Competitividad / EDU2015-70912C2-1-R. La web del proyecto se puede consultar en: $<$ https://esbrina. eu/es/portfolio/apren-do-como-aprenden-los-docentes-implicacioneseducativas-y-retos-para-afrontar-el-cambio-social/ $>$.

${ }^{8}$ Se trata de un proyecto coordinado y el grupo Elkarrikertuz del País Vasco se ocupa del profesorado de educación infantil y primaria.
} 
científicos, historiadores, etc.) - no mediante el estudio de cómo el alumnado aprende realmente.

- La forma de determinar el éxito de la escuela es examinar al alumnado para comprobar cuántos hechos y procedimientos han adquirido.

Sin embargo, esta forma entender el conocimiento escolar tan profundamente enraizada, naturalizada y persistente, incluso en la enseñanza superior, no puede responder a las necesidades presentes y futuras del alumnado actual. Y no puede porque no tiene en cuenta:

- Ni la diversidad social y cultural de los estudiantes actuales.

- Ni el desarrollo de la "economía del conocimiento", basada en "la producción y distribución del conocimiento y la información, más que en la producción y distribución de cosas" (DRUCKER, 1993, p. 182).

- Ni las nuevas formas de nuevas formas de producción, representación y transmisión y valoración del conocimiento (GIBBONS et al., 1994).

- Ni las implicaciones del ingente desarrollo de las Tecnologías de la Información y la Comunicación (SANCHO, 2010), ni el impacto de los multialfabetismos (COPE; KALANTZIS, 2000; KRESS, 2003).

De ahí la importancia de que una escuela de educación infantil, primaria o secundaria, una universidad o un sistema escolar que quiera plantearse una trasformación constante de su organización, gestión y práctica educativa, con el fin de ofrecer al alumnado la mejor educación posible en cada momento, tenga que preguntarse:

- Cuáles son las representaciones y concepciones actuales del conocimiento?

- Cómo se elabora, almacena, transmite, se accede y se valora?

- Qué concepciones y representaciones del conocimiento contribuyen a nuestro desarrollo como individuos, ciudadanos y miembros de sociedades justas, éticas y estéticas?

\section{LAS TECNOLOGÍAS EDUCATIVAS}

Las instituciones educativas están tan naturalizadas que no se tiende a considerar que no dejan de ser una creación, un dispositivo, como señalaba Foucault, ideado y desarrollado por seres humanos a través de un conjunto de tecnologías artefactuales, organizativas, simbólicas e incluso biotecnológicas (ÀLVAREZ et al., 1993). En este sentido, como señalaba (MECKLENBURGER, 1990, p. 106), "la escuela es una 'tecnología' de la educación del mismo modo que los coches son una 'tecnología' del transporte". Sin embargo, muchas personas se sorprenden de que un libro de texto sea una tecnología (artefactual y simbólica) de la información y de que la disposición del currículo constituya una potente tecnología simbólica y organizativa. Así como que los edificios y los equipamientos sean, a menudo, durísimas tecnologías artefactuales y simbólicas. Porque para muchas personas, parece que solo es "tecnología" todo lo vinculado con lo digital.

Valga como ejemplo la pregunta de una periodista a experto en innovación educativa, asesor del Gobierno británico: “¿Puede la tecnología sustituir a los libros de texto?". A lo que él contestó: "El problema es cómo los usamos. Si los profesores solo siguen el libro, se están perdiendo el elemento personal. Si todo lo que hacemos es cambiar los libros por tecnología y usarla del mismo modo se trata simplemente de otra forma de cometer el mismo error" (ÀLVAREZ, 2017). Una consideración que nos permita abrir dos líneas de pensamiento.

La primera se refiere a la dificultad que representa para la innovación y transformación de los sistemas escolares, no tener en cuenta los saberes, las habilidades, los propósitos y las visiones de la educación que los han ido constituyendo. Una situación que explica el difícil acceso que están teniendo unas las tecnologías cambiantes, versátiles y 'blandas' como las digitales, en un dispositivo tecnológico tan 'duro' y naturalizado como la Escuela (SANCHO, 1996). La segunda apunta a uno de los aspectos que forman parte del previsible fracaso de las innovaciones, al señalar que una innovación, menos una transformación educativa, no puede consistir en introducir un elemento nuevo mientras todo lo demás permanece igual (SANCHO; ALONSO, 2012).

De aquí que, a la hora de plantearnos una auténtica transformación institucional, no baste con preguntarse por los medios hoy dominantes de elaboración, almacenamiento, transmisión, acceso y valorización de la información, algo de por sí complejo por su multiplicidad y la 'agresividad' comercial de las multinacionales'. También habrá que analizar si los medios, de todo tipo, utilizados responden a las finalidades educativas planteadas.

\section{LAS EXPERIENCIAS DE APRENDIZAJE}

Suelo preguntarles a mis estudiantes del grado de Pedagogía, sobre las actividades/experiencias de aprendizaje que jalonaron su vida escolar, incluso universitaria. La mayoría dedicó prácticamente todo su tiempo escolar, incluida la Universidad, a escuchar, aplicar y repetir, de la forma más conveniente, lo

\footnotetext{
9 Ver la columna de opinión: No pongas tus... manos sobre la educación. Disponible en: <http://eldiariodelaeducacion.com/blog/2017/02/10/nopongas-tus-manos-sobre-la-educacion>. Acceso en: 11 feb. 2017.
} 
explicado por los docentes, los libros de texto o el material didáctico. Muy pocos han vivido experiencias de aprendizaje colaborativo, basadas en indagación, la resolución de problemas, la toma de decisiones, etc. Una situación que está en línea con la investigación llevada a cabo por Larry CUBAN, a principio de la década de 1990, sobre los cambios y las continuidades en las aulas de Estados Unidos entre 1890 y 1990). Un estudio que le lleva a la conclusión de que, a pesar de las reformas y las ideas innovadoras, "enseñar es decir, aprender es escuchar y el conocimiento el contenido de los libros de texto" (CUBAN, 1993, p. 27).

En estos momentos tenemos evidencias de que se aprende con sentido, de que no olvidamos todo una vez pasado el examen, cuando se contextualiza lo que se aprende; cuando se aprende en colaboración; cuando se permite y fomenta dirigir la propia indagación; cuando se logra que nos sorprendamos por su propio aprendizaje; cuando se piensa y se actúa en una red de relaciones; cuando se puede trasladar lo aprendido a nuevas situaciones (ATKINSON, 2011; DARLIN-HAMMOND, 2008; HERNÁNDEZ, F.; SANCHO, 2016 2016; STOLL; FINK; EARL, 2004, entre otros 10).

De ahí que, en el proceso continuado de transformación y mejora de la enseñanza, otro tema fundamental sea: ¿Qué experiencias de enseñanza y aprendizaje pueden propiciar procesos de aprendizaje genuinos y significativos que promuevan la curiosidad, la creatividad, la responsabilidad y la pasión por el aprendizaje? Y no sólo por parte de los estudiantes, sino también de los docentes.

\section{LA EVALUACIÓN}

Uno de los elementos que suele producir más turbulencias ante cualquier intento de innovar o transformar la enseñanza es la evaluación del aprendizaje. Cómo asegurar que el alumnado haya aprendido lo que se pretendía? Al final, a pesar de las muchas críticas recibidas y de las consecuencias que pueden tener para los estudiantes, los docentes y las escuelas, el recurso más utilizado sigue siendo la prueba de papel y lápiz (CARABAÑA, 2015; NICHOLS; BERLINER, 2007). Un recurso que no da cuenta de lo que cada uno ha aprendido, sino de su capacidad o habilidad para contestar en un momento dado lo que se le pregunta y de la forma que quien lo hace ha previsto como correcta.

A pesar de contar con numerosas propuestas alternativas para la evaluación (BOALER, 2016 GRIFFIN; CARE, 2014; HERNÁNDEZ, F. et al., 2011;

\footnotetext{
${ }^{10}$ Ver Cuadernos de Pedagogía, 483, nov. 2017. Tema del mes "Hazlo tú mismo, piensa por ti mismo".
}

SANCHO, 1998b; 2013; entre otros), por razones muy diferentes (tiempo, formación, recursos, dificultades para salir de la 'zona de confort', etc.), difícilmente las encontramos instauradas en las aulas. El profesorado, además de, en general, no sentirse seguro ni respaldado por la implementación de nuevos modos de evaluación, suele alegar que los estudiantes están muy acostumbrados al enfoque tradicional de la enseñanza y que son los primeros en rechazar, o ver con suspicacia, los cambios educativos. Los estudiantes tienden a sospechar del cambio cuando no se sienten involucrados o autorizados y cuando temen que al final serán evaluados de acuerdo a lo que los docentes (o quienes desarrollen las pruebas) consideren aprendizaje, en lugar de preguntarles lo que realmente han aprendido. Esta situación llevó al profesor de Harvard y el MIT Judah Schwartz - ante la iniciativa School of the 21st Century impulsada por la administración de George H. W. Bush a comienzos de la década de 1990 -, a platear que los estudiantes necesitan involucrarse en cualquier iniciativa de cambio desde el principio y que la evaluación ha de ser uno de los primeros temas a tratar (SCHWARTZ; KENNEY, 2008). Porque sin un cambio profundo de las formas de evaluar, es difícil o imposible lograr transformaciones significativas.

De aquí que la última y no menos importante cuestión a tratar sea ¿cuáles son los sistemas de evaluación que nos permiten mínimamente garantizar que podamos hablar sobre lo que el alumnado ha aprendido, y no sólo sobre lo que ha hecho o ha conseguido recordar (o copiar)?

\section{CONCLUSIONES}

Los profundos cambios políticos, económicos, sociales, culturales y tecnológicos acaecidos en los últimos cuarenta años están teniendo un impacto considerable en las instituciones educativas. Unas instituciones centradas en la tradición que hoy no solo tienen que preservar el pasado, sino contribuir a crear el presente y el futuro.

La historia de la educación también es la de las ideas y propuestas innovadoras de un conjunto de personas comprometidas y visionarias. Pero, al mismo tiempo, la de una inercial institucional que dificulta transformaciones significativas, sostenibles y con sentido de la equidad social.

En los últimos años se han multiplicado las presiones para que las instituciones educativas innoven y se adapten a las necesidades de la sociedad de la información. Pero la Escuela no es solo una empresa (aunque cada día esté más privatizada) sino que puede representar un papel fundamental para contribuir a la equidad y la armonía social. Aunque para que esto suceda, no solo se ha de estar a la moda pedagógica y al dictado de las multinacionales digitales. Sino comprometerse en 
una agenda de transformación en la que se tendrá que discutir una y otra vez sobre quienes son los docentes y los estudiantes y sus modos de aprender; sobre la noción de conocimiento; sobre la naturaleza y función de las tecnologías educativas; sobre las experiencias de aprendizaje y sobre los modos de evaluar todo el proceso y los resultados de la enseñanza y el aprendizaje.

El camino no parece fácil, y no lo es, porque no hay recetas, no hay fórmulas mágicas. Se trata de una perspectiva educativa, una visión política y pedagógica, que además ha de contar con las distintas facetas que constituyen cada contexto. Sí, una tarea apasionante, a la vez que difícil. Pero como argumentaba Seneca, el filósofo de la Roma Antigua, "no nos atrevemos a muchas cosas porque son difíciles, pero son difíciles porque no nos atrevemos a hacerlo". Este artículo es una invitación a que nos atrevamos seguir intentándolo.

\section{REFERENCIAS}

ADAMS BECKER, Samantha; CUMMINS, Michele; DAVIS, A., et al. NMC Horizon Report: 2017 Higher Education Edition. Austin, Texas: The New Media Consortium, 2017. Disponible en: <https://www.nmc.org/publication/nmchorizon-report-2017-higher-education-edition/>. Acceso en: 15 sept. 2017.

ÁLVAREZ, Àlvar; MARTÍNEZ, Antonio; MÉNDEZ, Roberto. Tecnología en acción. Barcelona: Rialp, 1993.

ÁlVAREZ, Pilar. Richard Gerver: "Hay demasiadas modas pasajeras en la educación”. EI País, 4 oct. 2017. Disponible en: <https://politica.elpais.com/politica/2017/10/02/actualidad/ 1506943955_129970.html>. Acceso en: 4 oct. 2017.

ANGUITA, Marisol; HERNÁNDEZ, Fernando; VENTURA, Montserrat. Los proyectos, tejido de relaciones y saberes. Cuadernos de Pedagogía, Barcelona, n. 400, p. 77-80, 2010.

ATKINSON, Dennis. Art, equality and learning. Pedagogies against the state. Rotterdam: Sense Publishers, 2011.

AUTORÍA COMPARTIDA. La moda de los proyectos. Cuadernos de Pedagogía, Barcelona, n. 467, p. 82-87, 2016.

BELASCO, James A. Enseñar a bailar al elefante. Barcelona: Plaza \& Janes, 1992.

BIESTA, Gert J. J. Beyond learning: democratic education for a human future. New York; London: Routledge, 2015.

BOALER, Jo. Mathematical mindsets: unleashing students' potential through creative math, inspiring messages and innovative teaching. San Francisco: Jossey-Bass Publishers, 2016.

BRENNAN, John; SHAH, Tarla (Ed.). Higher education and society in changing times: looking back and looking forward. Cheri. Open University, 2011. Disponible en: <https://www. open.ac.uk/cheri/documents/Lookingbackandlookingforward. pdf $>$. Acceso en: 2 mar. 2013.

BUXARRAIS, Rosa M.; MARTÍNEZ, Miquel (Eds.). Retos educativos para el siglo XXI. Autonomía, responsabilidad, neurociencia y aprendizaje. Barcelona: Octaedro, 2015.
CARABAÑA, Julio (2015). La inutilidad de PISA para las escuelas. Madrid: Catarata, 2015.

COPE, Bill; KALANTZIS, Mary. Multiliteracies. Literacy learning and the design of social futures. London; New York: Routledge, 2000.

CUBAN, Larry. How teachers taught: constancy and change in American classrooms, 1890-1990. New York: Teachers College Press, 1993.

DARLIN-HAMMOND, L. (Coord.). Powerful learning. What we know about teaching for understanding. San Francisco, CA: Jossey-Bass, 2008.

DRUCKER, Peter F. The rise of the knowledge society. The Wilson Quarterly, v.17, n. 2, p. 52- 71, Spring, 1993.

EDU-FACTORY Y UNIVERSIDAD NÓMADA (Comps.). La universidad en conflicto. Capturas y fugas en el mercado global del siglo del saber. Madrid: Traficantes de Sueños, 2010.

ERSTAD, Ola; KUMPULAINEN, Kristiina; MÄKITALO, et al. (Eds.). Learning across contexts in the knowledge society. Rotterdam/Boston/Taipei: Sense Publishers, 2016.

FOUCAULT, Michael. Vigilar y castigar: Nacimiento de la prisión. Madrid: Siglo XXI, 1976.

FOUCAULT, Michael. Dits et ecrits. Tome III. París: Gallimard, 1994.

FULLAN, Michael. Los nuevos significados del cambio en la educación. Barcelona: Octaedro, 2002.

GIBBONS, Michael; LIMOGES, Camille; NOWOTNY, Helga et al. La nueva producción del conocimiento: la dinámica de la ciencia y la investigación en las sociedades contemporáneas. Barcelona: Pomares-Corredor, 1997.

GRIFFIN, Patrick; CARE, Esther (Ed.). Assessment and teaching of 21st century skills. Methods and Approach. Dordrecht: Springer Netherlands.

HEGEL, Georg W. F. Ciencia de la lógica. Buenos Aires: Solar, 1974 (3. ed.) [1812-1816].

HERNÁNDEZ, Fernando (Coord.). ¡Y luego dicen que la escuela pública no funciona! Investigar con los jóvenes sobre cómo transitan y aprenden dentro y fuera de los centros de secundaria. Barcelona: Octaedro, 2017.

HERNÁNDEZ, Fernando; FORÉS, Anna; SANCHO, Juana M. et al. Aprender desde la indagación en la Universidad. Barcelona: Octaedro-ICE-UB. Cuadernos de docencia universitaria, 19, 2011.

HERNÁNDEZ, Fernando; SANCHO, Juana M. (Coord.). La perspectiva DIY en la universidad: ¡hazlo tú mismo y en colaboración! Barcelona: Octaedro, 2016.

HERNÁNDEZ, Fernando; VENTURA, Montserrat (2008). Prólogo a la presente edición. In: HERNÁNDEZ, Fernando; VENTURA, Montserrat. La organización del currículum por proyectos de trabajo. El conocimiento es un calidoscopio. Barcelona: Octaedro, 2008.

HERNÁNDEZ, José M. La renovación pedagógica en España al final de la transición. El encuentro de los movimientos de renovación pedagógica y el ministro Maravall (1983). Educación i História: Revista d'História de l'Educación, n. 18, p. 81-105, 2011. 
ITO, Mizuko; BAUMER, Sonja; BITTANTI, Matteo et al. Hanging out, messing around, and geeking out: kids living and learning with new media. Cambridge: MIT Press, 2010.

JUUSO, Hannu; LINDH, Anja; HASARI, Matti et al. Researchbased school and teacher education: publication of Finnish teacher training schools 2014. Oulu: University of Oulu, University Oulu University Teacher Training School, 2014.

KRESS, Gunther. Literacies in the new media age. London: Routledge, 2003.

LIVINGSTONE, Sonia; SEFTON-GREEN, Julian. The class. Living and learning in the digital age. New York: New York University Press, 2016.

MCCLINTOCK, Robert O. El alcance de las posibilidades pedagógicas. In: MCCLINTOCK, Robert; VÁZQUEZ, Gonzalo; STREIBEL, Michael J. (Org.). Comunicación, tecnología y diseños de instrucción: la construcción del conocimiento escolar y el uso de los ordenadores. Madrid: Cide-MEC. 1993. p. 104-125.

MECKLENBURGER, James A. Educational technology is not enough. Phi Delta Kappan, p. 104-108, Oct. 1990.

MIÑO, Raquel. Trajectòries d'aprenentatge através de contextos. Dos estudis de cas sobre els trànsits dels joves entre el dins i el fora de l'escola secundària. Barcelona: Universidad de Barcelona. Tesis doctoral no publicada, 2017.

MIÑO, Raquel; ALONSO, Cristina; BOSCO, Alejandra, et al. Geografías e historias de aprendizaje de docentes de secundaria. Intersecciones, transiciones y zonas de no saber. VI Jornadas de historias de vida. Lérida, España, 17-18 de oct. 2017.

MONTGOMERY, Anthony; KEHOE, Ian (Eds.). Reimagining the purpose of schools and educational organisations. Developing critical thinking, agency, beliefs in schools and educational organisations. Springer International Publishing, 2016 .

NICHOLS, Sharon L.; BERLINER, David. Collateral damage: how high-stakes testing corrupts America's schools. Cambridge, MA: Harvard Education Press, 2007.

PHILLIPS, D. C. Research in the hard sciences, and in very hard "softer" domains. Educational Researcher, Washington, v. 43, n. 1, p. 9-11, 2014. 10.3102/0013189X13520293

RIGOLON, Alessandro. European design types for 21st century schools: an overview. CELE Exchange, Centre for Effective Learning Environments, 2010/03, OECD Publishing, Paris. Disponible en: <http://dx.doi. org/10.1787/5kmh36gpvmbx-en>. Acceso 15 jun. 2011.

SANCHO, Juana M. Software educativo. Los límites duros de una tecnología blanda. IV Jornadas de Software educativo. Granada: Centro de Profesores de Granada, 1996, p. 53-70.

SANCHO, Juana M. El ilusionismo legislativo: un acercamiento a la reforma del sistema educativo español. Paideia - Revista de la Univerisdad de Concepción, Chile, n. 25, p. 31-51, 1998 a.

SANCHO, Juana. M. El portafolio: la evaluación como reconstrucción del proceso de trabajo. In: BIQUERRA, Rafael; ÁlVAREZ, Manuel (Coord.). Manual de orientación y tutoría. Barcelona: Praxis, 1998b, p. 463-464.

SANCHO, Juana M. Diversificar los espacios de enseñanza. Cuadernos de Pedagogía, Barcelona, n. 290, p. 54-57, 2000.
SANCHO, Juana M. Digital Technologies and Educational Change. In: HARGREAVES, Andy; LIEBERMAN, Ann; FULLAN, Michael et al. (Eds.). Second International Handbook of Educational Change. Springer International Handbooks of Education, 2010. p. 433-444.

SANCHO, Juana M. Una propuesta para valorar el trabajo de los estudiantes más allá de los formatos técnicos. In: PAREDES, Joaquín; HERNÁNDEZ, Fernando; CORREA, José M. (Eds.). La relación pedagógica en la universidad, lo transdisciplinar y los estudiantes. Desdibujando fronteras, buscando puntos de encuentro. Madrid: Depósito digital UAM <http://hdl. handle.net/10486/13152>, 2013, p. 66-84.

SANCHO, Juana M.; ALONSO, C. (Coord.). La fugacidad de las políticas, la inercia de las prácticas. La educación y las tecnologías de la información y la comunicación. Barcelona: Octaedro, 2012.

SANCHO, Junana M.; HERNÁNDEZ, Fermando (Coord.). Tecnologias para transformar a educação. Porto Alegre (Brasil): ArtMed, 2006.

SANCHO, Juana M.; HERNÁNDEZ, Fernando (Org.). Professores na incerteza: aprender a docência no mundo atual. Porto Alegre: Penso, 2016.

SANCHO, Juana M.; HERNÁNDEZ, Fernando; CARBONELL, Jaume et al. Aprendendo com as inovações nas escolas. Porto Alegre, Brasil: ArtMed, 2000.

SARASON, Seymour B. The predictable failure of educational reform: can we change course before it's too late? San Francisco: Jossey-Bass, 1990.

SAWYER, R. Keith. Optimising learning: implications of learning sciences research. Paris. OECD, 2008.

SCHWARTZ, Judah L.; KENNEY, Joan M. Tasks and rubrics for balanced mathematics assessment in primary and elementary grades. Thousand Oaks, CA: Corwin Press, 2008.

SENGE, Peter. La quinta disciplina. El arte y la práctica de la organización abierta al aprendizaje. Barcelona: Granica, 1990.

STOLL, Louise; FINK, Dean, EARL, Lorna. Sobre el aprender y el tiempo que requiere. Barcelona: Octadro, 2004.

STOLL, Louise; FINK, Dean. Para cambiar nuestras escuelas. Barcelona: Octaedro, 1999.

SWANGER, Dustin. Innovation in higher education: can colleges really change? 2016. Disponible en: <https://www. fmcc.edu/about/files/2016/06/Innovation-in-Higher-Education. pdf>. Acceso en: 14 feb. 2017.

THOMAS, Douglas; BROWN, John S. A new culture of learning: cultivating the imagination for a world of constant change. Lexington, Ky: CreateSpace?, 2011.

TWENGE J. IGen: Why today's super-connected kids are growing up less rebellious, more tolerant, less happy and completely unprepared for adulthood - and what that means for the rest of us. New York: ATRIA Books, 2017.

Recebido em 24-12-2017

Aprovado em 01-03-2018. 